\title{
Effects of dipotassium glycyrrhizinate on wound healing
}

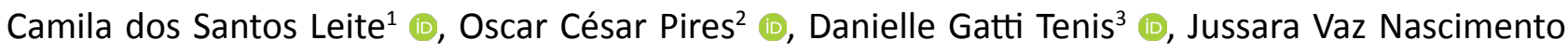 \\ Ziegler $^{4} \mathbb{D}$, Denise Gonçalves Priolli ${ }^{5} \mathbb{D}$, Thalita Rocha ${ }^{6 *}$
}

1. MSc, Fellow PhD degree. Multidisciplinary Research Laboratory, Universidade São Francisco (USF) - Bragança Paulista (SP), Brazil.

2. PhD, Assistant Professor. Physiology and Pharmacology Laboratory - Universidade de Taubaté (UNITAU) - Taubate (SP), Brazil.

3. Graduate student. Universidade São Francisco (USF) - Bragança Paulista (SP), Brazil.

4. MSc. Multidisciplinary Research Laboratory - Universidade São Francisco (USF) - Bragança Paulista (SP), Brazil.

5. PhD, Assistant Professor. Multidisciplinary Research Laboratory - Universidade São Francisco (USF) - Bragança Paulista (SP), Brazil.

6. PhD, Assistant Professor. Multidisciplinary Research Laboratory - Universidade São Francisco (USF) - Bragança Paulista (SP), Brazil.

\begin{abstract}
Purpose: Dipotassium glycyrrhizinate (DPG) has anti-inflammatory properties, besides promoting the regeneration of skeletal muscle. However, it has not been reported on skin wound healing/ regeneration. This research aimed to characterize the effects of DPG in the treatment of excisional wounds by second intention. Methods: Male adults $(n=10)$ and elderly $(n=10)$ Wistar rats were used. Two circular wounds were excised on the dorsal skin. The excised normal skins were considered adult (GAN) and elderly (GIN) naïve. For seven days, 2\% DPG was applied on the proximal excision: treated adult (GADPG) and elderly (GIDPG), whereas distal excisions were untreated adult (GANT) and elderly (GINT). Wound healing areas were daily measured and removed for morphological analyses after the $14^{\text {th }}$ and the $21^{\text {st }}$ postoperative day. Slides were stained with hematoxylin-eosin, Masson's trichrome, and picrosirius red. Results: Histological analysis revealed intact (GAN/GIN) and regenerated (GANT/GINT/GADPG/GIDPG) skins. No differences of wounds' size were found among treated groups. Epidermis was thicker after 14 days and thinner after 21 days of DPG administration. Higher collagen I density was found in GIDPG (14 ${ }^{\text {th }}$ day) and GADPG (21 $1^{\text {st }}$ day). Conclusion: DPG induced wound healing/skin regeneration, with collagen I, being more effective in the first 14 days after injury.
\end{abstract}

Key words: Glycyrrhizic Acid. Wound Healing. Collagen. Models, Animal. Rats.

*Corresponding author: tharocha@yahoo.com | (55 19)98189-7545

Received: Apr 09, 2021 | Review: Jun 11, 2021 | Accepted: July 08, 2021

Conflict of interest: Nothing to declare.

Research performed at Multidisciplinary Research Laboratory, Universidade São Francisco, Bragança Paulista (SP), Brazil. 


\section{Introduction}

Wound healing is characterized by cellular and molecular complex events, consisting of three phases-inflammatory, proliferative and remodelling-, and involves cell division, reepithelialization, neovascularization, collagen synthesis, remodelling and structural contraction ${ }^{1,2}$.

The healing mechanism occurs physiologically, but there are several local and/or systemic factors that can interfere negatively, for example: contamination, smoking, inadequate treatment, oxygen supply and deficient nutrients due to decreased perilesional vascularity, immunosuppressive disease, medications (corticosteroids, chemotherapy, radiotherapy) and nutritional deficiencies (vitamins $B$, $C, D$ and $E$ ), and aging, regarding the individual's clinical conditions and chronic diseases, such as cardiovascular diseases, diabetes mellitus and associated neuropathies ${ }^{3-7}$.

Biological aging is a process in which important physiological, morphological, and biochemical changes gradually occur. Loss of the arrangement between the elastic and collagen fibers in the dermal papillae or decrease in size and proliferation of keratinocytes in the basal stratum lead to epidermis thickness structural and functional changes, clearly identifiable in the elderly skin $^{5}$. Specifically, the dermis can undergo progressive changes, with a thin thickness due to the fibroblasts rarefaction, collagen synthesis decreasing and elastic fibers fragmentation. Also, changes in the amount of water and glycosaminoglycans occur, creating spaces between elastic and collagen fibers. In addition, cell migration capability and vascularity reduce, and size and secretion of sebaceous and sweat glands decrease $e^{5}$. Those structural changes result in a thin and fragile skin in elderly, vulnerable to mechanical forces and contamination, as well as in a slow process of skin healing ${ }^{5,7}$.

In recent years, research has been carried out seeking for alternative methods or drugs to help, correct, improve, or accelerate the skin healing process. Thus, new compounds have been studied, aiming mainly to promote regeneration of the epidermis ${ }^{8}$ and dermis ${ }^{8-10}$.
Glycyrrhiza glabra L., known as licorice, has shown nutritional and pharmacological properties especially assigned to glycyrrhizin ${ }^{11}$. Another isolated compound, the glycyrrhizic acid (GA), has been used regarding its antitumor, antiallergic, antibiotic and anti-inflammatory properties $^{12-14}$. Dipotassium glycyrrhizinate (DPG), a secondary product of GA, has antiallergic, antibiotic and anti-inflammatory effects similar to the corticosteroids' ones, but without side effects, as skin allergic reactions ${ }^{15,16}$. On cells, DPG is able to inhibit the hyaluronidase enzyme and, consequently, avoid the damage to the extracellular matrix, the histamine release and inflammatory chemical mediators, leukotrienes and prostaglandins ${ }^{16}$.

Experimental study using DPG on skeletal muscle pointed to its regenerative capacity, suggesting that DPG is able to modulate the satellite cells, induce myoblast and myotube differentiation and lead to muscle hyperplasia ${ }^{17}$. On glioblastoma cells, DPG controls tumor proliferation by inducing apoptosis through the inhibition of the nuclear factor kappa B (NF-kB), related to inflammation, mediated signaling pathway ${ }^{18}$.

Considering the cellular and molecular complexity mechanisms involved in the skin wound healing, the physiological and structural changes in the elderly skin that can contribute to the delay in repair, and the DPG properties, this research attempted to characterize its benefits in skin wound healing, through assessment the re-epithelization, changes in epidermis and dermis thickness, identification and quantification of dermic collagens I and III comparing adult and elderly skin over the 21 days of healing in animal model of excisional healing.

\section{Methods}

\section{Dipotassium glycyrrhizinate cream}

DPG $\left(\mathrm{C}_{42} \mathrm{H}_{60} \mathrm{~K}_{2} \mathrm{O}_{16}\right)$ was manipulated as a cream at the concentration of $2 \%$ (Table 1 ). It was supplied by Verdi Cosmetics (Joanopolis-SP, Brazil, 64.786.031/0001-00).

Table 1 - Dipotassium glycyrrhizinate cream gel $2 \%$.

\begin{tabular}{ccccc} 
Ingredient & $\%$ & Function & INCI Name & CAS Number \\
\hline Aqua & 93.600 & Solvent & Aqua & $7732-18-5$ \\
Sepigel 305 & 4.000 & Viscosity controlling & $\begin{array}{c}\text { Polyacrylamide, C13-14 } \\
\text { Isoparaffin (and) Laureth } 7\end{array}$ & $\begin{array}{c}\text { 9003-05-8/246538-79-4/68439-50- } \\
9 / 9002-92-0 / 7732-18-5\end{array}$ \\
$\begin{array}{c}\text { Dipotassium } \\
\text { glycyrrhizinate }\end{array}$ & 2.000 & Active & Dipotassium glycyrrhizinate \\
Euxyl PE 9010 & 0.400 & Preservative & $\begin{array}{c}\text { Phenoxyethanol (and) } \\
\text { Ethylhexylglycerin }\end{array}$
\end{tabular}

INCI: International Nomenclature of Cosmetics Ingredients; CAS: Chemical Abstracts Service. 


\section{Experimental groups}

The research was previously approved by the Ethical Committee for Animal Use (CEUA) of Universidade São Francisco (protocol no. 001.09.17) and Universidade de Taubaté (protocol no. 005/17), following the guidelines of the Brazilian Society of Laboratory Animal Sciences (SBCAL).

Wistar rats were supplied by Laboratory Animals Breeding and Commerce (ANILAB Laboratório de Diagnóstico Animal, Paulínia, SP, Brazil) and kept at the Animal Experimentation Vivarium of Universidade de Taubaté in individual cages, at $22 \pm 3^{\circ} \mathrm{C}$ on a $12 \mathrm{~h} \mathrm{light/dark}$ cycle, with free access to standard diet and ad libitum water.

Twenty male rats (Wistar) were used-10 adult animals ( 3 months old, weighing between $274-390 \mathrm{~g}$ ) and 10 elderly animals (12 months old, weighing between 454-569 g).

\section{Surgical procedure}

The animals were submitted to anesthetic procedure with $2 \%$ xylazine hydrochloride (Xilazin ${ }^{\circledR}$, Syntec, Santana de Parnaíba, SP, Brazil) (10 mg. $\left.\mathrm{kg}^{-1}\right)$ associated with $5 \%$ dextrocetamine hydrochloride (Ketamin ${ }^{\circledR}$, Cristália, Itapira, $\mathrm{SP}$, Brazil) $\left(25 \mathrm{mg} \cdot \mathrm{kg}^{-1}\right)$, prepared by combining $0.5 \mathrm{~mL}$ of xylazine (10 mg) with $0.5 \mathrm{~mL}$ of ketamine $(25 \mathrm{mg}$ ) to a volume of $1 \mathrm{~mL}$ administered by intraperitoneally $\left(1 \mathrm{~mL} . \mathrm{kg}^{-1}\right)^{8}$.

After trichotomy of the dorsal region and skin asepsis, each animal was submitted to two circular excisions, with $2 \mathrm{~cm}$ in diameter, $2 \mathrm{~cm}$ distant from each other, in median dorsal plane, limited in depth by muscle aponeurosis, performed with a scalpel (handle and blade number 15$)^{8}$.

The experiment was randomized into naïve (health skin), untreated (NT) or DPG-treated (DPG) animals and divided into groups following site (proximal or distal), treatment (untreated or DPG-treated) and day of euthanasia (14 or 21). After excision, the proximal wound was untreated, and distal wound was daily treated with $0.1 \mathrm{~mL}$ of $2 \%$ DPG cream, for seven days, starting 24 hours after surgery. Both proximal (untreated) and distal wound (DPG-treated) did not require bandages and healed by second intention, based on literature ${ }^{8}$, which used tretinoin for the same purpose. All animals were euthanized after the wound scar excisions at day 14 or 21, as described in Fig. 1.

- $\operatorname{GAN}(n=10)$ : the skin initially removed from adult (A) was used as naïve (N) group;

- GIN $(n=10)$ : the skin initially removed from elderly (I) was used as naïve (N) group;

- GA14 ( $n=5$ ): skin (proximal and distal) from GAN (adult) group submitted to excisional scar in vivo at day 14. Those skin originated the groups GANT14 (untreated, proximal skin, euthanasia at day 14) and GADPG14 (DPG-treated, distal skin, euthanasia at day 14);
- GA21 ( $=5$ ): skin (proximal and distal) from GAN (adult) group submitted to excisional scar in vivo at day 21. This skin originated the groups GANT21 (untreated, proximal skin, euthanasia at day 21) and GADPG21 (DPG-treated, distal skin at day 21);

- GI14 ( $=5$ ): skin (proximal and distal) from GIN (elderly) group and submitted to excisional scar in vivo at day 14. Those skin originated the groups GINT14 NT (untreated, proximal skin, euthanasia at day 14) and GIDPG14 (DPG-treated, distal skin euthanasia at day 14);

- $\mathrm{GI} 21(\mathrm{n}=5)$ : skin (proximal and distal) from GIN (elderly) group and submitted to excisional scar in vivo at day 21. This skin originated the groups GINT21 (untreated, proximal skin, euthanasia at day 21) and GIDPG21 (DPG-treated, distal skin euthanasia at day 21).

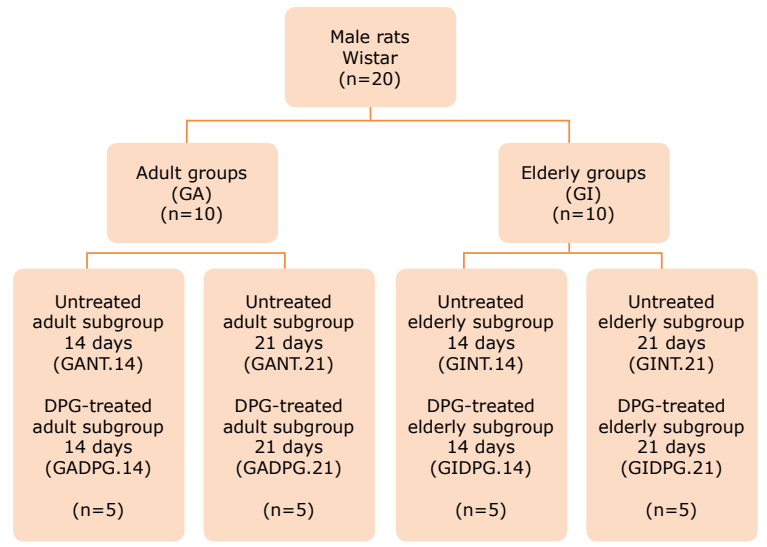

Figure 1 - Fluxogram of experimental groups: adults $(G A)$; elderly (GI); non-treated-GANT14, GANT21, GINT14, and GINT21; treated-GADPG14, GADPG21, GIDPG14, and GIDPG21.

\section{Macroscopic analysis}

The DPG-treated and untreated wound healing in both adult and elderly animals were evaluated throughout the experimental period, comparing the groups by macroscopy ${ }^{8}$. The initial area (large versus small diameter) was measured from day 0 to day 21 using a caliper (Universal Digimess 100003, Sao Paulo, SP, Brazil) and the scar tissue was removed on day $14^{8}$ (GA14: GANT14, GADPG14; GI14: GINT14, GIDPG14) or on day 218 (GA21: GANT21, GADPG21; GI21: GINT21; GIDP21).

\section{Microscopic analysis}

For microscopical analysis, the skin samples were fixed in $10 \%$ formaldehyde solution (Labsynth ${ }^{\circledR}$, Diadema, SP, Brazil) for $24 \mathrm{~h}$, attached at the end to cork, dehydrated in increasing ethanol concentrations (Labsynth ${ }^{\circledR}$, Diadema, SP, Brazil), clarified in xylol (Labsynth ${ }^{\circledR}$, Diadema, SP, 
Brazil), embedded in paraffin (Labsynth ${ }^{\circledR}$, Diadema, SP, Brazil), and submitted to microtomy (Lupetec MRPO3, São Carlos, SP, Brazil).

The slides (sections $5-\mu \mathrm{m}$ thick) were deparaffinized in two xylol baths (10 minutes each), hydrated in decreasing series of ethanol $(100,95,80$, and $70 \%)$ and distilled water, and stained with hematoxylin-eosin (HE) to measure epidermis thickness. Masson's trichrome (MT) was used to measure dermis thickness, and picrosirius red (PR) to identify and quantify collagen fibers. After staining, slides were dehydrated in an increasing series of ethanol 170 , 80,95 , and $100 \%$ ) and xylol, and mounted with synthetic Canada balsam.

Epidermis, dermis thickness and collagen quantification were measured by computer-assisted image analysis method ${ }^{8,19,20}$ under an optical microscope. The image capture system consisted of a digital color camera Infinite-3C ${ }^{\circledR}$ (Lumenera, Ottawa, Canada) coupled to a microscope Nikon ${ }^{\circledR}$ a-photo-2-YSC (Nikon, Tokyo, Japan) connected to a computer (Dell ${ }^{\circledR}$, Austin, United States; Pentium 4 processor, dual-core, $1.8 \mathrm{Mb}$, Windows $\mathrm{XP}^{\circledR}$ platform). Final magnification of 40x was used for epidermis and of $10 x$ for dermis and collagen fibers. From each histological section, three different fields were analyzed. The images were sent and processed on a computer using the NIS for Windows program ${ }^{8}$.

\section{Statistical analysis}

The analysis of the results was performed by adopting $p<0.05$, to reject the null hypothesis using the following statistical tools: sample size, descriptive statistics, measures of central tendency, normality test, comparison test ( $t$ test) to homoscedastic distribution or Mann-Whitney to heteroscedastic distribution; ANOVA followed by Bonferroni post-hoc tests, partial correlation (Spearman's test). The Statistical Package for the Social Sciences (SPSS) 20.0 (IBM Corporation, São Paulo, SP, Brazil) for Windows was used.

\section{Results}

Histological analysis revealed intact skins for GAN and GIN. In both groups, epidermis presented a stratified squamous epithelium, with stratum basalis (basal cell layer), stratum spinosum (prickle cell layer), stratum granulosum (granular cell layer), stratum lucidum, stratum corneum (keratin layer), and dermis (papillary and reticular dermis) composed of connective tissue consisting of collagen containing blood vessels, hair shafts and glands, considering the specific aspects of an adult skin and an elderly one ${ }^{5}$, in which the arrangement between collagen fibers in the dermis is lost and the proliferation of keratinocytes in the stratum basalis decreases. After the experimental protocol, it is possible to observe the progressive closure of the excisional area, in adults and elderly animals, after 7, 14 and 21 days (Fig. 2).

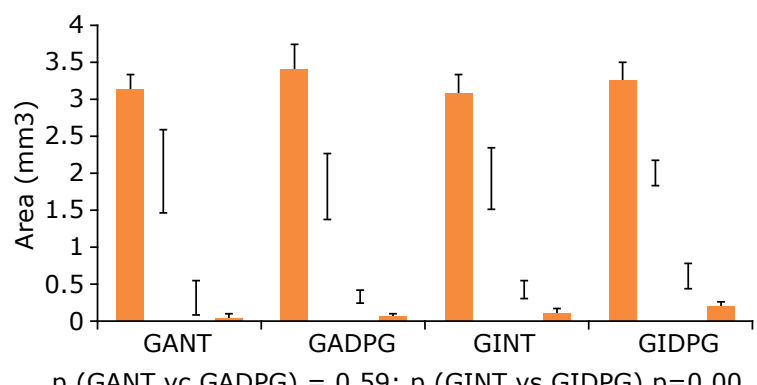

$p($ GANT vc GADPG) $=0,59 ; p$ (GINT vs GIDPG) $p=0.00$

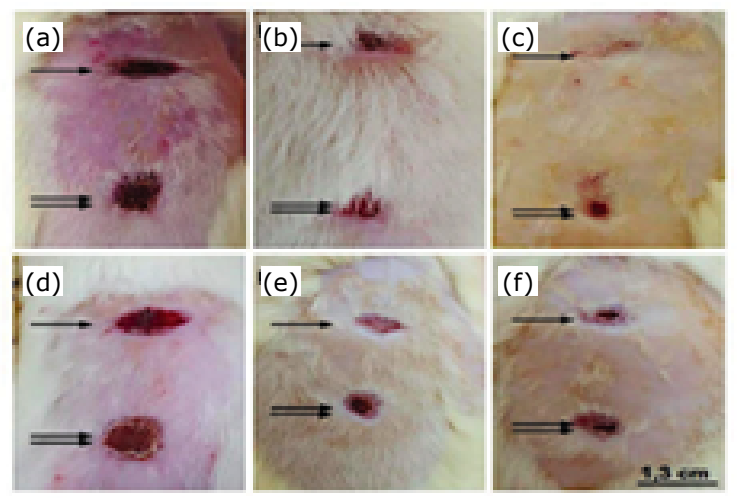

GANT: non-treated group adult naïve; GADPG: group adult treated with DPG; GINT: non-treated group elderly nontreated; GIDPG: group elderly treated with DPG; DPG: dipotassium glycyrrhizinate.

Figure 2 - Graphical representation (groups GANT, GADPG, GINT and GIDPG; area in $\mathrm{mm}^{3}$ ) and macroscopical analysis (a-f) of wound healing overtime of skin resection [after $7(a, d), 14$ (b, e), 21 (c, f) days]. Observe the macroscopic aspect in adults $(n=10)$ (a-c) and elderly ( $n=10$, Bonferroni post-hoc test) (d-f) animals, untreated (arrow) and treated (double-arrow) with DPG (bar=1.3 cm).

The epidermis thickness of the regenerated skin, in adults and elderly animals, after 14 days, was similar between GANT14/GADPG14, and GINT14/GIDPG14. Regarding DPG effects in the regenerated skin, epidermis was thicker after DPG administration in adult and elderly rats when compared to naïve (GAN/GIN) skins (Fig. 3). For dermis, there were no significant changes in collagen I in adult animals, treated (GADPG) or untreated (GANT) (Fig. 4). However, higher collagen I density was observed in elderly animals, especially in those treated with DPG (GIDPG). Elderly animals, untreated or treated with DPG, decreased collagen III distribution $(p=0.027)$ (Figs. 5 and 6). 

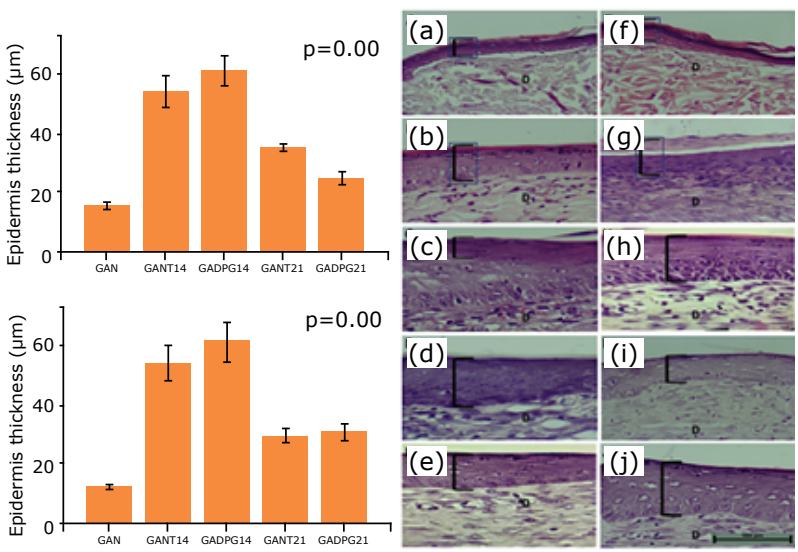

GAN: group adult naïve; GANT: non-treated group adult naïve; GADPG: group adult treated with DPG; GIN: group elderly; GINT: non-treated group elderly; GIDPG: group elderly treated with DPG; DPG: dipotassium glycyrrhizinate.

Figure 3 - Graphical representation and histological analysis of epidermis thickness (groups GAN - a, GANT14 - b, GADPG14 - c, GANT21 - d, GADPG21 - e; GIN - f, GINT14 - g, GIDPG14 - h, GINT21 - $\mathbf{i}$ and GIDPG21 - j; bar $=100 \mu \mathrm{m})$. Observe the normal aspects in naïve $(\mathbf{a}, \mathbf{f})$ and regenerated (b-e; $\mathbf{g}-\mathbf{j})$ skins. Note the increase of epidermis thickness after DPG treatment in adult $(n=10)$, Bonferroni post-hoc test (c) and elderly $(n=10)$ $(h, j)$ animals.
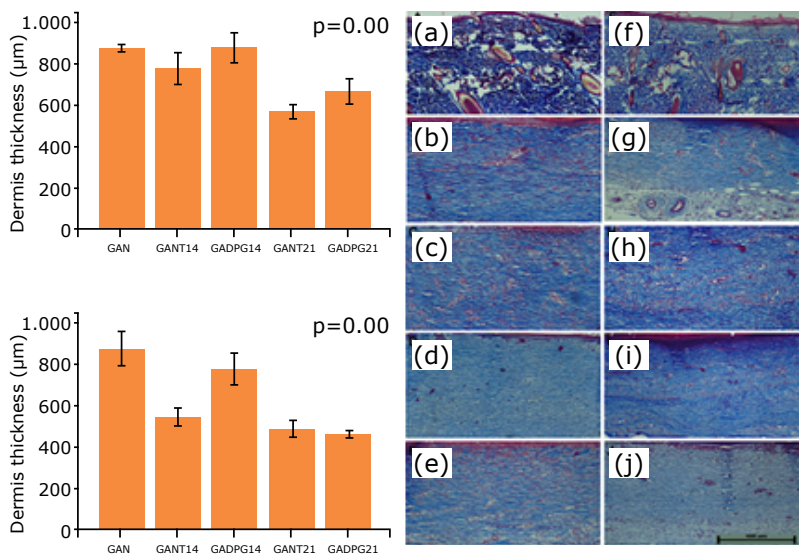

GAN: group adult naïve; GANT: non-treated group adult naïve; GADPG: group adult treated with DPG; GIN: group elderly; GINT: non-treated group elderly; GIDPG: group elderly treated with DPG; DPG: dipotassium glycyrrhizinate.

Figure 4 - Graphical representation and histological analysis of dermis thickness (groups GAN - a, GANT14 - $\mathbf{b}$, GADPG14 - c, GANT21 - d, GADPG21 - e; GIN - f, GINT14 - g, GIDPG14 - h, GINT21 - i and GIDPG21 - j; bar=100 $\mu \mathrm{m})$. Observe the normal aspects and collagen distribution in naïve $(\mathbf{a}, \mathbf{f})$ and regenerated (b-e; $\mathbf{g}-\mathbf{j})$ skins. Cutaneous appendages were not observed in regenerated dermis (b-e; g-j). (n=10, Dunnett post-hoc test).
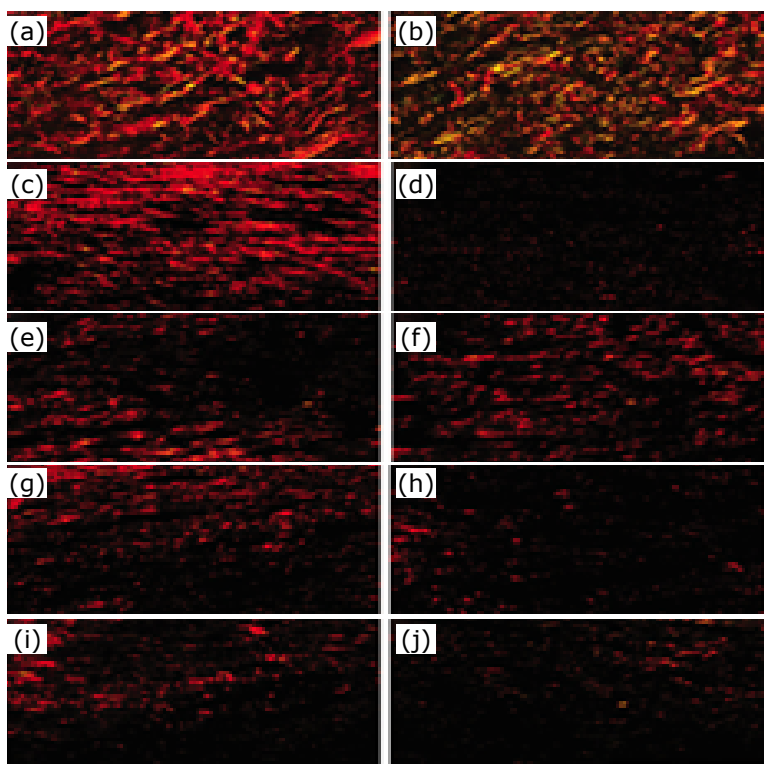

GAN: group adult naïve; GANT: non-treated group adult naive; GADPG: group adult treated with DPG; GIN: group elderly; GINT: non-treated group elderly; GIDPG: group elderly treated with DPG; DPG: dipotassium glycyrrhizinate.

Figure 5 - Graphical representation of collagens I (a, c) and III (b, d) distribution in the dermis of groups GAN, GANT14, GADPG14, GANT21, GADPG21, GIN, GINT14, GIDPG14, GINT21 and GIDPG21. Observe that the amount of collagen I is higher than the one of collagen III in adult (a) and elderly (c) animals. Note that the collagen III significantly reduced in (b) adult and elderly (d) animals after skin resection ( $n=10$, Dunnett post-hoc test). (a)

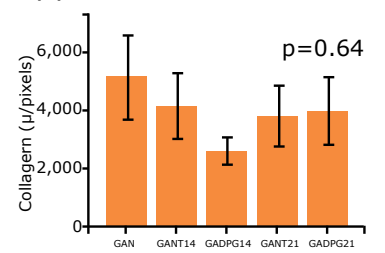

(c)

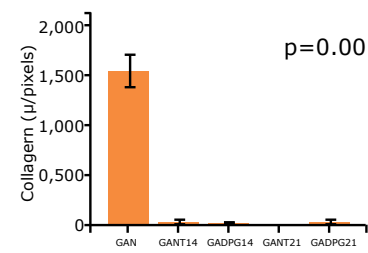

(b)

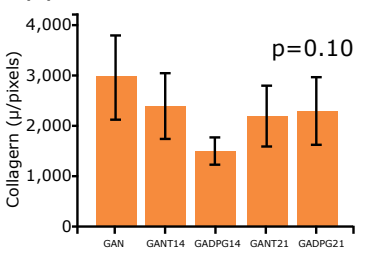

(d)

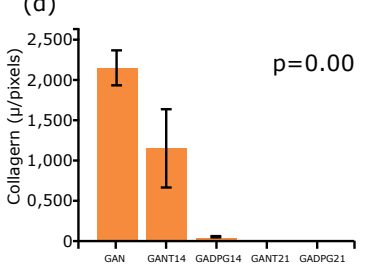

GAN: group adult naïve; GIN: group elderly; GANT: nontreated group adult naïve; GINT: non-treated group elderly; GADPG: group adult treated with DPG; GIDPG: group elderly treated with DPG; DPG: dipotassium glycyrrhizinate.

Figure 6 - Histological analysis of collagen I (red) and collagen III (green) distribution in the dermis of groups GAN (a), GIN (b), GANT14 (c), GINT14 (d), GADPG14 (e), GIDPG14 (f), GANT21 (g) GINT21 (h), GADPG21 (i) and GIDPG21 (j) (bar=100 $\mu \mathrm{m})$. ( $n=10$, Dunnett post-hoc test). 
Regenerated skins in adults and elderly animals, after 21 days, displayed the same histological aspects of naïve skins in GANT, GINT, GADPG, and GIDPG groups (Figs. 3 and 4). For regenerated skins, the epidermis thickness was similar between GANT21 and GADPG21, and was thicker in GANT21, GADPG21, GINT21, GIDPG21 when compared to naïve skin (Fig. 3). For dermis, higher collagen I density was observed in GADPG21 when compared to naïve skins (Fig. 4). There were no significant changes in collagen I in adult animals treated (GADPG21) when compared to untreated ones (GANT21) (Figs. 5 and 6).

Comparing the regenerated skins after 14 and 21 days, the epidermis thickness was similar between GANT14/ GADPG14, GINT14/GIDPG14, and GINT21/GIDPG21, being thicker after 14 days than after 21 days. Considering the epidermis of adult animals, there were significant changes in GAN vs. GANT14 ( $p=0.000)$, GAN vs. GADPG14 $(p=0.000)$, GAN vs. GANT21 ( $p=0.006)$, GAN vs. GADPG $21(p=0.006)$, and GADPG14 vs. GADPG21 $(p=0.000)$, although the epidermis was thicker in GANT21 than GADPG21 ( $p=0.02)$. For elderly animals, regarding epidermis, the difference was found when the groups GIN and GINT14 ( $p=0.001), G I N$ and GIDPG14 ( $p=0.001), \operatorname{GINT} 14$ and GINT21 ( $p=0.01)$, and GIDPG14 and GIDPG21 ( $p=0.001$ ) were compared (Fig. 3). Epidermis was thinner after 21 days of DPG administration.

Dermis was also restructured with papillary and reticular portions, but without skin appendages. It was thicker in GAN/GIN, when compared with treated or untreated dermis. After 14 days, dermis was thicker than after 21 days, especially in elderly animals $(p=0.005)$ when compared to adults. Adults' dermis thickness was similar among all groups. However, for elderly ones there were differences among GIN vs. GINT14 ( $p=0.001)$, GINT14 vs. GIDPG14 $(p=0.045)$, GIDPG14 vs. GIDPG21 ( $p=0.003$ ) groups (Fig. 4).

Under polarized light microscopy type I collagen, there were red-colored birefringence and type III collagen green birefringence. Type I collagen fibers were more abundant than type III collagen fibers in adult and elderly groups (Fig. 5).

There were no significant changes in collagen I in adult animals treated (GADPG) or untreated (GANT) at 14 and 21 days. However, adult animals showed differences on the collagen III when comparing the groups GAN vs. GANT14 $(p=0.000)$, GAN vs. GADPG14 ( $p=0.000)$, GAN vs. GANT21 $(p=0.000)$, and GAN vs. GADPG21 ( $p=0.000)$. In elderly animals, the amount of collagen I was similar between GIN and GIDPG14, but lower in GINT14. The opposite was observed after 21 days, when collagen I was higher in GINT21 than in GIDPG21. There was difference in collagen III among groups GIN vs. GIDPG14 ( $p=0.00)$, GIN vs. GINT21 ( $p=0.00)$, GINT14 vs. GIDPG14 ( $p=0.00)$, and GINT14 vs. GINT21 ( $p=0.026)$. After 14 days, elderly animals untreated or treated with DPG decreased collagen III distribution ( $p=0.027$ ) (Fig. 6).

There are some associations between variables in the present study, such as: age is inversely related to collagen I ( $r=-0.474, p<0.01)$, and collagen III is inversely correlated to day of evolution ( $r=-0.467, p=0.01)$.

The mean and standard deviation found in each group are presented in Table 2.

Table 2 - Epidermis and dermis thickness and collagens I and III quantification per experimental group*.

\begin{tabular}{|c|c|c|c|c|c|}
\hline Skin & Day & Group & Mean & $\begin{array}{l}\text { Standard } \\
\text { deviation }\end{array}$ & p-value \\
\hline \multirow{10}{*}{ Epidermis thickness $(\mu \mathrm{m})$} & \multirow{2}{*}{ do } & GAN & 15.24 & 2.52 & \multirow{10}{*}{0.000} \\
\hline & & GIN & 12.34 & 1.90 & \\
\hline & \multirow{4}{*}{$\mathrm{d} 14$} & GANT & 54.13 & 12.40 & \\
\hline & & GADPG & 61.39 & 10.82 & \\
\hline & & GINT & 54.09 & 14.19 & \\
\hline & & GIDPG & 61.95 & 14.92 & \\
\hline & \multirow{4}{*}{$\mathrm{d} 21$} & GANT & 35.60 & 2.92 & \\
\hline & & GADPG & 24.87 & 4.91 & \\
\hline & & GINT & 29.62 & 5.97 & \\
\hline & & GIDPG & 30.78 & 6.81 & \\
\hline \multirow{10}{*}{ Dermis thickness $(\mu \mathrm{m})$} & \multirow{2}{*}{ do } & GAN & 868.81 & 50.20 & \multirow{10}{*}{0.000} \\
\hline & & GIN & 1097.48 & 125.99 & \\
\hline & \multirow{4}{*}{ d14 } & GANT & 598.02 & 138.72 & \\
\hline & & GADPG & 816.83 & 312.76 & \\
\hline & & GINT & 649.21 & 130.86 & \\
\hline & & GIDPG & 934.26 & 214.33 & \\
\hline & \multirow{4}{*}{$\mathrm{d} 21$} & GANT & 566.46 & 74.19 & \\
\hline & & GADPG & 666.64 & 140.54 & \\
\hline & & GINT & 579.81 & 125.95 & \\
\hline & & GIDPG & 546.45 & 64.92 & \\
\hline
\end{tabular}


Table 2 - Continuation.

\begin{tabular}{|c|c|c|c|c|c|}
\hline Skin & Day & Group & Mean & $\begin{array}{l}\text { Standard } \\
\text { deviation }\end{array}$ & p-value \\
\hline \multirow{9}{*}{ Collagen I } & \multirow{2}{*}{ do } & GAN & 5.13 & 3.35 & \multirow{9}{*}{0.047} \\
\hline & & GIN & 2.65 & 1.56 & \\
\hline & \multirow{3}{*}{$\mathrm{d} 14$} & GANT & 4.10 & 2.69 & \\
\hline & & GADPG & 2.52 & 1.01 & \\
\hline & & GINT & 1.19 & 0.89 & \\
\hline & \multirow{4}{*}{$\mathrm{d} 21$} & GANT & 3.76 & 2.39 & \\
\hline & & GADPG & 3.89 & 2.93 & \\
\hline & & GINT & 1.35 & 0.80 & \\
\hline & & GIDPG & 0.60 & 0.30 & \\
\hline \multirow{9}{*}{ Collagen III } & \multirow{2}{*}{$\mathrm{do}$} & GAN & 1.53 & 0.41 & \multirow{9}{*}{0.000} \\
\hline & & GIN & 2.17 & 0.47 & \\
\hline & \multirow{3}{*}{$\mathrm{d} 14$} & GADPG & 0.008 & 0.009 & \\
\hline & & GINT & 1.16 & 1.10 & \\
\hline & & GIDPG & 0.01 & 0.017 & \\
\hline & \multirow{4}{*}{$\mathrm{d} 21$} & GANT & 0.0006 & 0.0005 & \\
\hline & & GADPG & 0.021 & 0.041 & \\
\hline & & GINT & 0.0001 & 0.0001 & \\
\hline & & GIDPG & 0.004 & 0.005 & \\
\hline
\end{tabular}

*Data are reported as means \pm standard deviation (analysis of variance); d0: day of excisional lesion; d14 and d21: euthanasia; GAN: group adult naïve; GIN: group elderly naïve; GANT: non-treated group adult; GADPG: group adult treated with DPG; GINT: nontreated group elderly; GIDPG: group elderly treated with DPG; DPG: dipotassium glycyrrhizinate.

\section{Discussion}

The healing process is a physiological response to an injury and is marked by cell proliferation of the basal stratum of the epidermis and re-epithelization ${ }^{1-4}$ collagens I and III synthesis s,21 $^{4}$ and dermal remodeling ${ }^{2,4,21}$.

In aging, important physiological and morphological events gradually occur, such as keratinocytes and fibroblasts apoptosis, collagen, elastin, proteoglycans and glycosaminoglycans synthesis decreation, dermal papillae shrinkage, thinner epidermis and dermis ${ }^{5,6}$, leading to a slow healing with functional and structural changes ${ }^{6,7}$.

In the last decades, research has been done to find alternative methods or new compounds that promote skin healing. Among them, retinoids, oils, and plant extracts have been studied. Such compounds were able to increase epidermis thickness ${ }^{8}$ and promote dermal tissue formation ${ }^{8-10}$.

Vegetable oils, such as Copaifera langsdorffii ${ }^{22}$ and Caryocar coriaceum Wittm ${ }^{23}$, Passiflora edulis extract (passion fruit) ${ }^{24}$ and Carissa spinarum Linn methanolic extract ${ }^{25}$, as well as DPG, were able to significantly accelerate the reduction of the injured area, increasing the rate of wound contraction and re-epithelization.

Such processes were observed after induced injury in the experimental groups studied. Specifically, when considering re-epithelialization of the affected area and epidermis thickness, it was effectively better in the groups treated with DPG. Differences in epidermal thickness are greater at 14 days than at 21 days in both adult and elderly treated animals. At 21 days, reduction in the thickness of the epidermis of the animals was observed at similar levels to the naïve animals.

The mechanism of re-epithelization by DPG is similar to the one of other compounds, such as leptin ${ }^{26}$, sericin ${ }^{27}$ and curcumin ${ }^{28}$, with significant proliferation, differentiation and migration of keratinocytes, increasing the thickness of the epidermis. Similar effect of DPG was observed in muscle regeneration studies, in which DPG induced differentiation of satellite cells in myoblasts and from these in myotubes, with formation of fully regenerated fibers early, after seven days ${ }^{17}$. These results suggest that DPG has a positive modulating effect on cellular hyperplasia, probably by activation of the NF-KB pathway, in a mechanism opposite to that one described for glioblastoma cells ${ }^{18}$.

Regarding the dermis, DPG induced the formation of a totally restructured extracellular matrix dermis, without signs of fibrosis. This is probably due to its inhibitory effect on the hyaluronidase enzyme and, consequently, on hyaluronic acid ${ }^{16}$.

Studies have shown that lipoic acid (LA ${ }^{29}, 18 \beta$-glycyrrhetinic acid $(18 \beta-G A)^{30}$ and tretinoin act significantly in the repair 
of the dermis, increasing the number of fibroblasts and the collagen synthesis ${ }^{8}$. Such results were also observed after the use of collagenase and trans-retinoic acid ${ }^{9,10}$, as well as DPG.

Collagen is the main structural protein produced by fibroblasts and makes up the extracellular matrix in normal tissue, becoming the most abundant protein during skin healing ${ }^{31}$. Normal dermis contains approximately $80-85 \%$ of collagen I and $10-15 \%$ of collagen III ${ }^{32,33}$. Collagen I are thick-stable fibers that guarantee the resistance of the tissue to mechanical forces, while collagen III are finefibrils, arranged below the basement membrane, that guarantee the adherence of the epidermis to the dermis ${ }^{34}$.

Skin is affected by photoaging, decreasing the levels of collagens I and || ${ }^{32,36}$, mucopolysaccharides and elastin and increasing the activity of collagenase ${ }^{35,36}$. In the skin healing process, collagen III synthesized during the proliferative phase is commonly replaced by collagen I during tissue remodeling phase ${ }^{37}$.

In this study, the presence of collagen I was observed in adult and elderly animals, after 14 and 21 days, treated or not with DPG. The amount of collagen I was higher in adult animals than in elderly animals, as expected. However, after 14 days, the effect of DPG on the dermis of elderly animals led to an increase in collagen I density to levels similar to the ones of naïve animals.

Increases in collagen III density levels were found in the deep dermis in hypertrophic scars ${ }^{38}$ and keloid scars ${ }^{39}$, diverging from the present findings, in which type III collagen density was reduced even in naïve animals and was even lower in all other experimental groups. The associations between age and collagen I and collagen III and day of evolution suggest decreasing of collagen type I occurs because of the age, and there is reduction of collagen II in wound healing process.

Comparing the evolution of healing between adult and elderly animals over the healing period ( 0 to 21 days), and considering the pharmacological properties of DPG, the compound acted during healing, accelerating the regenerative process, especially in elderly animals. The results show a possible therapeutic potential of DPG, for the pharmaceutical and cosmetic industry, in wound healing by second intention, leading to re-epithelization and formation of a new dermis, rich in type I collagen.

\section{Conclusions}

DPG promoted more effective epidermis and dermis proliferation and hypertrophy in the first 14 days, including the period of use (first seven days), in adult and elderly animals. Its continuous application could improve healing. Regarding collagen I synthesis, it was higher in adults, after 21 days, and in elderly, after 14 days, in detriment to the synthesis of collagen type III, without inducing fibrosis.
The results suggest that DPG rebuilds dermis in a way similar to undamaged conditions.

\section{Author's contribution}

Conception and design of the study: Rocha T; Acquisition of data: Leite CS and Pires OC; Interpretation of data: Priolli DG; Analysis and interpretation of data: Rocha T; Technical procedures: Leite CS, Tenis DG and Ziegler JVN; Statistics analysis: Priolli DG; Manuscript preparation and writing: Leite CS; Critical revision: Pires OC.

\section{Data availability statement}

Data will be available upon request.

\section{Funding}

Not applicable.

\section{Acknowledgments}

Not applicable.

\section{References}

1. Rodrigues M, Kosaric N, Bonham CA, Gurtner GC. Wound healing: a cellular perspective. Physiol Rev. 2019;99(1):665706. https://doi.org/10.1152/physrev.00067.2017

2. Sorg H, Tilkorn DJ, Hager S, Hauser J, Mirastschijski U. Skin wound healing: an update on the current knowledge and concepts. Eur Surg Res. 2017;58(1-2):81-94. https://doi.org/10.1159/000454919

3. Anderson K, Hamm RL. Factors that impair wound healing. J Am Coll Clin Wound Spec. 2014;4(4):84-91. https://doi. org/10.1016/j.jccw.2014.03.001

4. Guo S, Dipietro LA. Factors affecting wound healing. J Dent Res. 2010;89(3):219-29. https://doi.org/10.1177/0022034509359125

5. Oriá RB, Ferreira FVA, Santana EN, Fernandes MR, Brito GAC. Study of age-related changes in human skin using histomorphometric and autofluorescence approaches. An Bras Dermatol. 2003;78(4):425-34. https://doi.org/10.1590/ S0365-05962003000400004

6. Bonifant $\mathrm{H}$, Holloway $\mathrm{S}$. A review of the effects of ageing on skin integrity and wound healing. Br J Community Nurs. 2019;24(3):2833. https://doi.org/10.12968/bjcn.2019.24.Sup3.S28

7. Baroni ERV, Simões MLPB, Auersvald A, Auerswald LA, Netto MRM, Simões RB, Ortolan MCAB, Kohler JN. Influence of aging on the quality of the skin of white women: the role of collagen. Acta Cir Bras. 2012;27(10):736-40. https://doi. org/10.1590/s0102-86502012001000012

8. Peseto DC, Carmona EV, Silva KC, Guedes FRV, Hummel Filho F, Martinez NP, Pereira JA, Rocha T, Priolli DG. Effects of tretinoin on wound healing in aged skin. Wound Rep Reg. 2016;24(2):411-7. https://doi.org/10.1111/wrr.12417 
9. Chang P, Guo B, Hui Q, Liu X, Tao K. A bioartificial dermal regeneration template promotes skin cell proliferation in vitro and enhances large skin wound healing in vivo. Oncotarget. 2017;8(15):25226-25241. https://doi. org/10.18632/oncotarget.16005

10. Pourjafar M, Saidijam M, Mansouri K, Ghasemibasir H, Dermani FK, Najafi R. All-trans retinoic acid preconditioning enhances proliferation, angiogenesis and migration of mesenchymal stem cell in vitro and enhances wound repair in vivo. Cell Prolif. 2017;50(1):12315. https://doi.org/10.1111/cpr.12315

11. Batiha GES, Beshbishy AM, El-Mleeh A, Abdel-Daim MM, Devkota HP. Traditional uses, bioactive chemical constituents, and pharmacological and toxicological activities of Glycyrrhiza glabra L. (Fabaceae). Biomolecules. 2020;10(3):pii:E352. https://doi.org/10.3390/biom10030352

12. Richard SA. Exploring the pivotal immunomodulatory and anti-inflammatory potentials of glycyrrhizic and glycyrrhetinic acids. Mediators Inflamm. 2021;(7):6699560. https://doi.org/10.1155/2021/6699560

13. Salem MMNM, Mohammed AAW, Al-Tameemi JA, Sulaiman GM. Biological study of the effect of licorice roots extract on serum lipid profile, liver enzymes and kidney function tests in albino mice. Afr J Biotechnol. 2011;10(59):12702-6. https://doi.org/10.5897/AJB11.1399.

14. Pastorino G, Cornara L, Soares S, Rodrigues F, Oliveira MBPP. Liquorice (Glycyrrhiza glabra): a phytochemical and pharmacological review. Phytother Res. 2018;32(12):232339. https://doi.org/10.1002/ptr.6178

15. Shim JM, Yim SB, Chung JH, Hong KS. Antiplaque and antigingivitis effects of a mouthrinse containing cetylpyridinium chloride, triclosan and dipotassium glycyrrhizinate. J Period Implant Sci. 2012;42(2):33-8. https://doi.org/10.5051/ jpis.2012.42.2.33

16. Vitali R, Palone F, Cucchiara S, Negroni A, Cavone L, Constanzo M, Aloi M, Dilillo A, Stronati M. Dipotassium glycyrrhizate inhibits HMGB1-dependent inflammation and ameliorates colitis in mice. PloS One. 2013;8(6):e66527. https://doi.org/10.1371/journal.pone.0066527

17. Rocha T, Bueno TOC, Nascimento L, Ziegler J, Gracia M, Priolli DG. Glycyrrhizinate dipotassium (DPG), myonecrosis control and skeletal muscle differentiation: a new adjuvant therapy for snake envenomation. FASEB J. 2017;31:622. https://doi.org/doi.org/10.1096/ fasebj.31.1_supplement.lb622

18. Bonafé GA, Santos JS, Ziegler JV, Umezawa K, Ribeiro $\mathrm{ML}$, Rocha T, Ortega MM. Growth inhibitory effects of dipotassium glycyrrhizinate in glioblastoma cell lines by target microRNAs through the NF-k B signaling pathway. Front Cell Neurosci. 2019;13(216):1-14. https://doi. org/10.3389/fncel.2019.00216

19. Priolli DG, Margarido NF, Martinez CA, Rotta CM, Stephani $\mathrm{SM}$. Edema quantification by computerized morphometry as an evaluation parameter for the resistance of colon anastomoses. Acta Cir Bras. 2003;18(5):398-406. https:// doi.org/10.1590/S0102-86502003000500005
20. Nonose R, Spadari AP, Priolli DG, Máximo FR, Aires Pereira $J A$, Martinez CAR. Tissue quantification of neutral and acid mucins in the mucosa of the colon with and without fecal stream in rats. Acta Cir Bras. 2009;24(4):105-11. https:// doi.org/10.1590/S0102-8650200900040000

21. Kiwanuka E, Junker J, Eriksson E. Harnessing growth factors to influence wound healing. Clin Plastic Surg. 2012;39(3):23948. https://doi.org/10.1016/j.cps.2012.04.003

22. Estevão LRM, Medeiros JP, Baratella-Evêncio L, Simões RS, Mendonça FS, Evêncio-Neto J. Effects of the topical administration of copaiba oil ointment (Copaifera langsdorffii) in skin flaps viability of rats. Acta Cir Bras. 2013;28(12):863-9. https://doi.org/10.1590/S0102-86502013001200009

23. Oliveira ML. Nunes-Pinheiro DC, Tomé AR, Mota EF, Lima-Verde IA, Campello CC, De Morais SM. In vivo topical anti-inflammatory and wound healing activities of the fixed oil of Caryocar coriaceum Wittm seeds. J Ethnopharmacol. 2010;129(2):214-9. https://doi. org/10.1016/j.jep.2010.03.014

24. Soares RDF, Campos MGN, Ribeiro GP, Salles BCC, Cardoso NS, Ribeiro JR, Souza RM, Leme KC, Soares CB, de Oliveira $C M$, Elston LB, da Fonseca CCP, Ferreira EB, Rodrigues MR, Duarte SMS, Paula FBA. Development of a chitosan hydrogel containing flavonoids extracted from Passiflora edulis leaves and the evaluation of its antioxidant and wound healing properties for the treatment of skin lesions in diabetic mice. J Biomed Mater Res A. 2020;108(3):65462. https://doi.org/10.1002/jbm.a.36845

25. Sanwal R, Chaudhary AK. Wound healing and antimicrobial potential of Carissa spinarum Linn in albino mice. J Ethnopharmacol. 2011;135(3):792-6. https://doi. org/10.1016/j.jep.2011.04.025

26. Tadokoro S, Ide S, Tokuyama R, Umeki H, Tatehara S, Kataoka S, Satomura K. Leptin promotes wound healing in the skin. Plos One. 2015;10(3):e0121242. https://doi. org/10.1371/journal.pone.0121242

27. Ersel M, Uyanikgil Y, Karbek AF, Ozcete E, Altunci YA, Karabey F, Cavusoglu T, Meral A, Yigitturk G, Cetin EO. Effects of silk sericin on incision wound healing in a dorsal skin flap wound healing rat model. Med Sci Monit. 2016;1(22):106478. https://doi.org/10.12659/MSM.897981

28. Kulac M, Aktas C, Tulubas F, Uygur R, Kanter M, Erboga $M$, Ceber M, Topcu B, Ozen OA. The effects of topical treatment with curcumin on burn wound healing in rats. J Mol Histol. 2013;44(1):83-90. https://doi.org/10.1007/s10735-012-9452-9

29. Külkamp-Guerreiro IC, Souza MN, Bianchin MD, Isoppo M, Freitas JS, Alves JA, Piovezan AP, Pohlmann AR, Guterres SS. Evaluation of lipoic acid topical application on rats skin wound healing. Acta Cir Bras. 2013;28(10):708-15. https:// doi.org/10.1590/S0102-86502013001000004

30. Hung CF, Hsia OCY, Hsieh WH, Li HJ, Tsai YJ, Lin CN, Chang $\mathrm{HH}, \mathrm{Wu}$ NL. 18ß-glycyrrhetinic acid derivative promotes proliferation, migration and aquaporin-3 expression in human dermal fibroblasts. Plos One. 2017;12(8):0182981. https://doi.org/10.1371/journal.pone.0182981 
31. Kallis PJ, Friedman AJ. Collagen powder in wound healing. J Drugs Dermatol. 2018;17(4):403-8.

32. El-Domyati M, Attia S, Saleh F, Brown D, Birk DE, Gasparro, F. Intrinsic aging vs. photoaging: a comparative histopathological, immunohistochemical, and ultrastructural study of skin. Exp Dermatol. 2002;11(5):398-405. https://doi.org/10.1034/j.16000625.2002.110502.x

33. Campos ACL, Branco AB, Groth AK. Wound healing. Arq Bras Cir Dig. 2007;20(1):51-8. https://doi.org/10.1590/ S0102-67202007000100010

34. Alves $A P N N$, Verde MEQL, Júnior $A E C F$, Silva $P G B$, Feitosa VP, Júnior EML, Miranda MJB, Filho MOM. Avaliação microscópica, estudo histoquímico e análise de propriedades tensiométricas da pele de tilápia do Nilo. Rev Bras Queimaduras. 2015;14(3):203-10.

35. Quan T, Shao Y, He T, Voorhees JJ, Fisher GJ. Reduced expression of connective tissue growth factor (CTGF/CCN2) mediates collagen loss in chronologically aged human skin. J Invest Dermatol. 2010;130(2):415-24. https://doi. org/10.1038/jid.2009.224
36. Varani J, Dame MK, Rittie L, Fligiel SEG, Sewon Kang S, Fisher GJ, Voorhees JJ. Decreased collagen production in chronologically aged skin. Roles of age-dependent alteration in fibroblast function and defective mechanical stimulation. Am J Pathol. 2006;168(6):1861-78. https:// doi.org/10.2353/ajpath.2006.051302

37. Ribeiro FAQ, Carvalho MFP, Pereira CSB, Tateno DA. Analysis of the concentration of collagen type I and III in the wound of the rat's skin treated with Mitomycin C. Arq Med Hosp Fac Cienc Med Santa Casa São Paulo. 2015;60:22-6.

38. Oliveira GV, Hawkins HK, Chinkes D, Burke A, Tavares AL, Ramos-e-Silva M, Albrecht TB, Kitten GT, Herndon DN. Hypertrophic versus non hypertrophic scars compared by immunohistochemistry and laser confocal microscopy: type I and III collagens. Int Wound J. 2009;6(6):445-52. https://doi.org/10.1111/j.1742-481X.2009.00638.x

39. Syed F, Ahmadi E, Iqbal SA, Singh S, McGrouther DA, Bayat A. Fibroblasts from the growing margin of keloid scars produce higher levels of collagen I and III compared with intralesional and extralesional sites: clinical implications for lesional sitedirected therapy. Br J Dermatol. 2011;164(1):83-96. https:// doi.org/10.1111/j.1365-2133.2010.10048.x 